Marcela Poučová

\title{
Sous les pavés, le noir! Le roman noir dans la France post-68
}

Brno: Masarykova univerzita 2017, 206 p.

Pavla DoležAlová [399@mail.muni.cz]

Masarykova univerzita, République tchèque

HTTPS://DOI.ORG/10.5817/ERB2019-2-22

Doté d'une couverture en noir et blanc ponctuée de rouge, le livre de Marcela Poučová porte le titre Sous les pavés, le noir! Le roman noir dans la France post68. La réalisation graphique dans le style de bande dessinée et la paraphrase du slogan célèbre des événements de Mai 68 préfigurent un des apports de cette étude: présenter un genre auparavant « mineur » et marginalisé au sein de la grande histoire de la littérature française, lui trouver une place sérieuse et le faire valoir.

Tandis que par leur devise "Sous les pavés, la plage! », les révolutionnaires voulaient rendre honneur à la découverte de la liberté sous la surface solide et pétrifiée de la vie bourgeoise, Poučová y voit autre chose, le noir. Il appartient d'ailleurs parmi les côtés estimables de son texte que " le noir » ainsi que maints autres termes habituellement utilisés pour traiter ce genre littéraire sont dès le début précisés dans le sous-chapitre 1.1.1 Quelques clarifications terminologiques. Forte d'un outil terminologique fiable, l'auteure peut procéder à un exposé méthodiquement divisé en six chapitres de longueur inégale, pourtant chacun répondant à la question centrale. Cet enjeu primaire, c'est l'image de la société française dans le miroir du roman policier, respectivement noir, paru dans la deuxième moitié du $\mathrm{XX}^{\mathrm{e}}$ siècle, notamment après 1968 . On découvre vite que cette image littéraire ne peut être, par définition, que déformée et partielle, pourtant le choix de l'histoire sociale en tant que « chair» du roman noir est sufisamment typique de la méthode créatrice des auteurs du genre en question. Une fois reconnu comme tel, ce genre relativement nouveau veut échapper aux qualificatifs tels que paralittérature, littérature populaire, littérature de gare etc. Sa « voie de légitimation » (chap. II et III) aurait ses propres stratégies et elle devrait être justifiée par les "pièces à conviction ", à savoir les prix littéraires, la presse spécialisée, les salons littéraires, les éditions spécialisées, et les outils médiatiques qui représentent aujourd'hui un espace mondialisé en expansion continue. L'histoire du genre dès ses racines et ses précurseurs est expliquée dans les chapitres IV et V, ne franchissant pas la fronitière du XXI ${ }^{e}$ siècle à une exception près (Vargas, Salut et liberté!, 2002 [?] dans la bibliographie). La « grande histoire » de la France est traitée sur le même plan temporel (chap. VI) et cet aperçu historique est utile au lecteur pour bien comprendre la vision antagoniste du monde qu'adopte le roman noir français, vision attestée par les citations significatives des théoriciens du genre. Citons-en J. Pons de l'an 1997 (p. 71) : « Le roman noir est une écriure engagée et offensive parce qu'en exhibant les mécanismes qui expliquent le pourquoi des choses et des actes, il dénonce les procédures de mensonge, d’aliénation et de violence qui quadrillent notre espace social ». De là il est facile à croire Poučová dans son affirmation lapidaire que " $[\mathrm{e}] \mathrm{n}$ effet, en choisissant un auteur du roman noir français, le lecteur à la recherche de ses convictions politiques gauchistes ne se trompera jamais » (p. 72). On peut y dépister ce qui est appelé la réalité française par l'intermédiaire du roman policier, resp. du polar, et des ses composantes thématiques. Ce sont par exemple les femmes : fatales, 
fragiles ou bien belliqueuses ; la musique : le jazz, le rock ; la ville et la province, les milieux défavorisés etc. Il est un peu dommage qu'en tant qu'une des formes d'identification, la langue n'ait pas reçu un sous-chapitre synthétisant les traits caractéristiques retrouvables à travers les extraits. Parmi ses formes sont pourtant cités les jeux de mots, les allusions et les pastiches, la langue parlée.

Loriginalité de cette étude repose en ce qu'elle donne un régistre répertorié de l'histoire du genre noir en France, dès ses racines et ses inspirations jusqu'à l'aube du XXI ${ }^{e}$ siècle. La méthode d'avoir préparé et présenté un vaste corpus de textes commentés pour illustrer les tendances repérées paraît la bonne. L'index des auteurs facilite l'orientation. Une future édition pourrait profiter éventuellement d'une structure plus équilibrée et des prolongements éclairant les dévéloppements les plus récents du genre en question. Or cette étude mériterait sans doute d'être publiée également en langue tchèque, au grand profit du vaste public intéressé qui ne lit pas en français. 
